\title{
Distribución temporal de insectos asociados a maracuyá (Passiflora edulis Sims) en Matagalpa, Nicaragua 2016
}

\section{Temporary distribution of insects associated to maracuyá (Passiflora edulis Sims) in Matagalpa, Nicaragua 2016}

\author{
Edgardo Jiménez-Martínez ${ }^{1}$, Roberto Gabriel Montano Núñez², Edwin Joé Bustamante Maradiaga ${ }^{2}$ \\ ${ }^{1}$ PhD en Entomología, ORCID: https://orcid.org/0000-0003-1086-7380, edgardo.jimenez@ci.una.edu.ni, (505) 2263-2609, \\ Universidad Nacional Agraria \\ ${ }^{2}$ Ingeniero Agrónomo, graduado en la Universidad Nacional Agraria
}

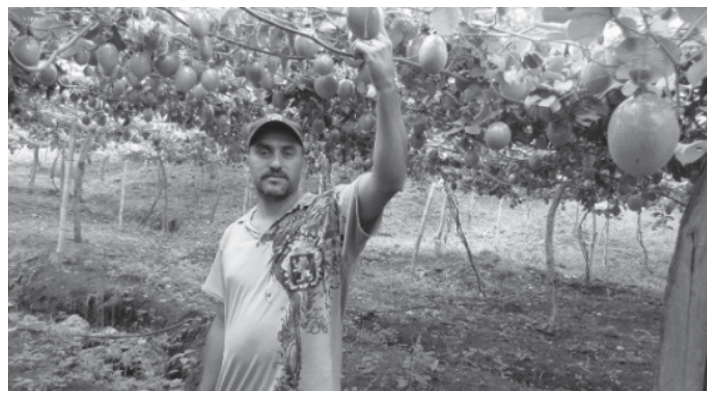

\section{RESUMEN}

La maracuyá (Passiflora edulis Sims) es una fruta tropical que pertenece a la familia de las Pasifloráceae en Nicaragua y se cultiva principalmente en Matagalpa, para el 2013, se reportan 310 fincas productoras de maracuyá. Con el propósito de contribuir al conocimiento científico nacional, se realizó este estudio entre julio a noviembre del 2016 en la comunidad Molino Sur Sébaco, Matagalpa, el propósito fue identificar, calcular la abundancia, riqueza, y fluctuación poblacional de las principales familias de insectos asociadas a la maracuyá. El estudio se desarrolló en dos fincas donde se colocaron 12 trampas por finca para insectos; además, en cada finca se realizó una colecta de 50 botones florales por fecha de muestreo para calcular el daño de la mosquita del botón floral. Las principales familias de insectos asociados al cultivo de maracuyá fueron las familias: Anthophoridae, Apidae, Coccidae, Curculionidae, Drosophilidae, Formicidae, Lonchaeidae, Noctuidae, Scarabaeidae y Vespidae. La diversidad insectil se estimó utilizando el índice de diversidad de Shannomweaver, la familia con mayor índice de diversidad fue la familia Drosophilidae. Se encontró que la mayor abundancia y riqueza de insectos asociados al cultivo de la maracuyá, fue encontrada en la finca Las Vegas. En cuanto a la riqueza de insectos se encontraron 32 familias y 41 géneros, siendo los más numerosos pertenecientes a las familias Apidae, Vespidae, Noctuidae, Pentatomidae y Scarabaeidae. En la distribución temporal, las familias más importantes fueron, la Lonchaeidae, Anthophoridae, Coccidae y Formicidae, entre los principales hábitos alimenticios de las especies insectiles asociadas al cultivo de maracuyá se encontraron, nectarívoro, polinivoro, depredador, defoliador y saprófago.

Palabras clave: biodiversidad, plagas, fluctuación, índice, artrópodos.
The passion fruit (Passiflora edulis Sims) is a tropical fruit that belongs to the family of Passifloraceas, in Nicaragua this one grown mainly in Matagalpa, in the 2013, 310 farms producing passion fruit were reported. In order to contribute to the national scientific knowledge, a study was conducted between July to November 2016, in the community of Molino Sur, Sébaco, Matagalpa, the study was carried out on two maracuya farms, with the porpoise of identifying, calculating abundance, richness and describing the temporal variation of the main insect families associated to passion fruit. The study was conducted in two farms, where 12 insect traps per farm were placed, in addition, in each farm, a collection of 50 flowers per monitoring date was examined for the floral fly damage, according to the results, the main insect families associated to the passion fruit were the families: Anthophoridae, Apidae, Coccidae Curculionidae, Drosophilidae, Formicidae, Lonchaeidae, Noctuidae, Scarabaeidae and Vespidae. The insect diversity was estimated using the Shannom-weaver diversity index, the family with the highest diversity index was the Drosophilidae family. It was found that the greatest abundance and richness of insects associated with the cultivation of passion fruit was found in the farm Las Vegas. As for the richness of insects, thirty-two families were found, with forty-one genera, the most numerous belonging to the families Apidae, Vespidae, Noctuidae, Pentatomidae and Scarabaeidae. Regarding the temporal variation, the most important families were the Lonchaeidae, Anthophoridae, Coccidae and Formicidae, among the main nutritional habits of the insect species associated with the cultivation of passion fruit were found, nectarivore, polinivore, predator, defoliator y saprophagus.

Key words: Biodiversity, pests, fluctuation, index, arthropods
Recibido: 23 de octubre del 2019

Aceptado: 10 de marzo del 2020

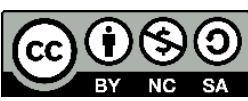

(C)
Los artículos de la revista La Calera de la Universidad Nacional Agraria, Nicaragua, se comparten bajo términos de la licencia Creative Commons: Reconocimiento, No Comercial, Compartir Igual. Las autorizaciones adicionales a las aquí delimitadas se pueden obtener en el correo edgardo.jimenez@ci.una.edu.ni Copyright 2020. Universidad Nacional Agraria (UNA) 


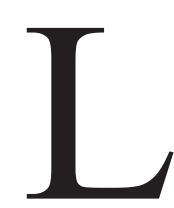

a maracuyá (Passiflora edulis Sims), es una fruta tropical que crece en forma de enredadera y que pertenece a la familia de las Pasifloráceas, de la que se conocen más de 400 variedades. Uno de los centro de origen de esta planta es Perú, presenta dos variedades o formas diferentes: la púrpura o morada ( $P$. edulis) y la amarilla ( $P$. edulis forma flavicarpa). La primera, principalmente, se consume en fresco y prospera en lugares semi cálidos y a mayor altura sobre el nivel del mar, en tanto que la segunda crece en climas cálidos, desde el nivel del mar hasta 1000 m de altitud. (Gerencia Regional Agraria La Libertad, 2009).

En Nicaragua la maracuyá se siembra principalmente en el departamento de Matagalpa en los municipios de Sébaco, San Ramón, Terrabona, San Dionisio, El Tuma- La Dalia y Matiguás, en este territorio las fincas se ubican entre los 450 a 1100 metros sobre el nivel del mar. Para el 2013 se reportaron 310 fincas productoras de maracuyá en los cuales se georreferenciaron aproximadamente 300 manzanas establecidas con este cultivo. Otros departamentos en donde se produce el cultivo pero en menos escala son: Rivas, Carazo, Masaya y Managua (Laguna et al., 2015).

La maracuyá es una buena fuente de proteínas, minerales, vitaminas, carbohidratos y grasas. Se consume cómo fruta fresca, en jugos y se utiliza para preparar gaseosas, néctares, yogurts, mermeladas, licores, pastelerías y confites. El jugo es el producto de mayor importancia, es una planta que se desarrolla en forma de enredadera ésta se adhiere a los soportes o tutores por medio de zarcillos que salen de las axilas de las hojas. Estas son alternas, profundamente trilobuladas, con márgenes finalmente dentados, miden de 7 a $20 \mathrm{~cm}$ de largo, y son de color verde profundo, brillante en el haz y más pálidas y sin brillo en el envés. Los tallos son ligeramente angulados cuando son jóvenes y cilíndricos en su etapa adulto de color verde claro a color verde oscuro (INTA, 1996).

Según López (2002) las plagas de la maracuyá que se han reportado en Nicaragua son ácaro rojo (Tetranychus sp, Bank), chinche patas de hoja (Leptoglossus zonatus Dallas), gusano defoliador (Dione juno Cramer), estos insectos causan daño a hojas, arrugamiento y caída de fruto en el cultivo de maracuyá. Estos Insectos se pueden controlar con métodos culturales, podas de tallos y ramas y con métodos químicos: uso de plaguicidas.

Según Bolaños, (1997) en Nicaragua también se han reportado cómo plagas de la maracuyá, nemátodo agalladores, que causa pudrición y deformación del sistema radicular y forma agallas, el gusano defoliador (D. Juno) devoran las hojas y causan defoliación completa, el chinche pata de hoja (L. zonatus) las ninfas y adultos succionan la savia de tallos, hojas, flores, frutos y causan arrugamiento del fruto, el chinche hediondo (Nezara viridula Linnaeus) ninfas y adultos succionan la savia, flores y frutos tiernos y causan la caída del fruto, los ácaros rojo (Tetranychus sp) y el ácaro blanco (Polyphagotarsonemus latus Bank) estos causan pérdida del color verde y caída de los hojas, también se desarrollan en hojas y brotes apicales nuevos.

En Nicaragua, los productores de maracuyá desconocen la diversidad y distribución temporal de los insectos que están presentes en este cultivo, por lo tanto, pocos saben distinguir entre insectos benéficos e insectos plagas. Ellos se encuentran con una serie de problemas al momento de realizar manejos, en el país existen pocos reportes formales sobre los principales órdenes, familias géneros y especies de insectos asociados al cultivo de maracuyá (Maes, 1998).

Debido a esta problemática, se realizó este estudio con el propósito de identificar los principales insectos asociados al cultivo de la maracuyá, con el propósito de conocer la diversidad, taxonomía e incidencia poblacional de los insectos asociados al cultivo de la maracuyá, finalmente se busca contribuir con nuevos conocimientos que pueden ser integrados a estrategias de manejo integrado de plagas en las plantaciones de maracuyá de Nicaragua.

\section{MATERIALES Y MÉTODOS}

Ubicación del estudio. El estudio se realizó entre los meses de julio a noviembre del 2016, en dos fincas experimentales con parcelas establecidas del cultivo de maracuyá amarillo (P. edulis forma flavicarpa). Las fincas están ubicadas en la comunidad Molino Sur, municipio de Sébaco departamento de Matagalpa el cual tiene una extensión territorial de 291 kilómetros cuadrados. Pósee una altitud de $470 \mathrm{msnm}$, en las coordenadas geográficas $12^{\circ} 50^{\prime} 5.16^{\prime \prime}$ latitud Norte y $85^{\circ} 59^{\prime}$ 41.74" longitud Oeste, la precipitación anual varía entre 800 y 2 $000 \mathrm{~mm}$ con temperatura promedio de $23.4^{\circ} \mathrm{C}$ (INETER, 2016).

La primera finca está ubicada en la comunidad Molino Sur, finca Las Vegas propiedad del Sr. Jarol Flores Arguello con una parcela de maracuyá de $3513 \mathrm{~m}^{2}$ con una edad del cultivo de un año. La segunda finca, también ubicada en la comunidad Molino Sur finca Linda Vista propiedad del Sr. Donis Alexander Flores Mercado con una parcela de maracuyá de $5269.5 \mathrm{~m}^{2}$, con una edad del cultivo de dos años. Ambas parcelas manejadas convencionalmente.

El diseño del estudio consistió, en la comparación de dos fincas. La cosecha se realizó cada ocho días, al igual que la aplicación de insecticidas. En cada finca se colocaron 12 trampas, seis de caída libre y seis de galón con melaza.

\section{Procesamiento de las muestras e identificación de insectos} en el laboratorio. Una vez recolectados los insectos en el campo, estos se llevaron al laboratorio de entomología y de plagas forestales de la Universidad nacional agraria, posteriormente se extrajeron los insectos de los viales entomológicos y se colocaron en platos petrix de $14 \mathrm{~cm}$ de 
ancho y dos cm de largo para ser lavados en alcohol al 75\%, luego cada muestra de insecto se colocó en papel toalla para que ellos se secaran durante 30 minutos a temperatura ambiente, en la identificación preliminar de los insectos, se utilizó estereoscopio marca ZEISS, Stemi 2000-C,de 10x $\mathrm{y}$ marca VANGUARD, de $2 \mathrm{x}$ y $4 \mathrm{x}$, para poder observar claramente las características morfológicas de las familias de insectos, además, se realizó comparación de especímenes con los del museo entomológico de la UNA y también se consultaron literaturas como Entomología Sistemática de Sáenz y De la Llana (1990) y Guía para el estudio de órdenes y familias de insectos de Centroamérica de Andrews y Caballero (1989), Entomología, Jiménez-Martínez (2009), entre otros libros de entomología.

Además de la revisión de literatura específica, se realizó una verificación preliminar de los especímenes por el Ing. Osvaldo Rodríguez de la UNA. Para el montaje de los insectos en cajas entomológicas, se procedió con la utilización de gradillas entomológicas, pinzas, pinceles, alfileres entomológicos número cuatro marca MORPHO de 38 y $45 \mathrm{~mm}$ de longitud, poroplas con una medición de $59 \mathrm{~cm}$ de ancho y de largo $121 \mathrm{~cm}$ y luego se ordenaron en las cajas entomológicas, cada insecto se rotulaba con dos etiquetas, la primer etiqueta llevaba los siguiente información (país, municipio, departamento y colector) la segunda etiqueta contenía el orden, la familia el género y la especie a la que pertenece el insecto.

\section{Variables evaluadas}

Abundancia total de insectos. Esta variable se registró desde el 8 de julio, una vez por semana hasta el 25 de noviembre 2016, se realizó un conteo de todos los insectos colectados en las trampas de caída libre y galones.

Abundancia total de insectos. Se realizó una sumatoria del total de insectos encontrados por tipos de trampas durante las fechas de colecta.

Abundancia de insectos de los principales órdenes. Se tomaron en cuenta los principales órdenes Díptera, Hymenoptera, Coleóptera, Lepidóptera y Hemíptera, se contaron el total de insectos en todas las fechas de colectas por tipo de trampa.

Comparación de la abundancia de insectos por familia. Se realizó un conteo de la abundancia de insectos de las diferentes familias encontradas en todas las fechas de colecta para determinar la finca con mayor número de familias.

Riqueza total de familias de insectos encontrados por finca. Se hizo un conteo del total de insectos por familias encontradas para determinar la finca con mayor riqueza de familias.
Riqueza total de géneros de insectos encontrados por finca. Se hizo un conteo por género de los insectos, para determinar la finca con mayor de riqueza de géneros.

\section{Índice de diversidad Shannon-Weaver de las principales} familias. Se comparó el índice de diversidad ShannonWeaver de las principales familias de insectos para medir la biodiversidad de insectos.

El índice de diversidad Shannon-Weaver es una medida importante para la biodiversidad. Este cálculo se realizó por cada finca.

1- Se encontró el número de familias dentro de la población de insectos.

2 -Se dividió el número de familia que observamos entre el número de la población para calcular la abundancia relativa.

3- Se calculó el logaritmo natural de la abundancia. Los cálculos logarítmicos los realizamos utilizando el botón Ln de la calculadora.

4-Se multiplicó la abundancia por el logaritmo natural de la abundancia. Esta es la suma de la abundancia y el logaritmo natural de la abundancia.

5- Se realizó una repetición de estos pasos para cada familia que se encontró en la toma de muestras.

6 -Se sumó el resultado de la abundancia y el logaritmo natural de la abundancia de cada familia.

7- Se multiplicó la cantidad calculada en el Paso 6 por -1. Esto es H'.

8-Se aumentó a la potencia de H'. Se calculó H 'en el paso 7. $\mathrm{Y}$ este fue nuestro índice de diversidad de Shannon-Weaver.

Formula que se utiliza para el cálculo de índice de diversidad de Shannon-Weaver.

$H^{\prime}=-\sum_{i=1}^{S} p_{i} \log _{2} p_{i}$

$\frac{n_{i}}{N}$

Dónde:

$\mathrm{S}=$ número de familias (la riqueza de familias)

$P i=$ proporción de individuos de la especie i respecto al total de individuos (es decir la abundancia relativa de la famillia $i$ ): $\mathrm{ni}=$ número de individuos de la familia $i$

$\mathrm{N}$ = número de todos los individuos de todas las familias

Distribución temporal de la mosquita del botón floral por finca. Se comparó la distribución temporal de insectos de la familia Lonchaeidae por fechas de colecta; se realizó la sumatoria del número de insectos en cada finca.

Distribución temporal de la familia Coccidae por finca. Se comparó la distribución de insectos de la familia Coccidae por 
fechas de colecta; se realizó sumatoria del número de insectos en cada finca.

Distribución temporal de la familia Coreidae por finca. Se comparó la distribución temporal de insectos de la familia Coreidae por fechas de colecta; se contabilizó el número de insectos en cada finca.

Distribución temporal de la familia Anthophoridae por finca. Se comparó la distribución temporal de insectos de la familia Anthophoridae por fechas de colecta y se realizó la sumatoria del número de insectos por finca.

Distribución temporal de la familia Formicidae por finca. Se comparó la distribución temporal de insectos de la familia Formicidae por fechas de colecta; se hizo la sumatoria del número insectos por finca.

Análisis de los datos. De acuerdo a la metodología utilizada por (Rugama y López, 2011, Lacayo y Mayorga 2014), después de colectados los insectos en el campo, estos fueron ordenados por variables de familias por finca en una tabla de datos en EXCEL, luego cada variable fue comparada entre fincas, utilizando un análisis de comparación de $t$ de Student, usando el programa de SAS (SAS, 2003). El nivel de significancia usado en el análisis fue de $(p=0.05)$ y se transformaron datos de $Y={ }^{2} \sqrt{X}$ para la familia Lonchaidae, con el objetivo de normalizar los datos, las bases de datos se normalizan para evitar la redundancia y proteger la integridad de los datos.

\section{RESULTADOS Y DISCUSIÓN}

Abundancia total de insectos. En el Cuadro 1, se presenta ocho órdenes de insectos, con 32 familias, 41 géneros y 41 especies, también se presenta el hábito alimenticio de cada orden, nectarívoro, defoliador, depredador, polinivoro, fitófago, parasitoide y saprófago de los insectos encontrados en las fincas Las Vegas y Linda Vista en el cultivo de maracuyá.

Abundancia total de insectos por finca. La abundancia total de insectos encontrados en trampas de caída libre y galones, en las dos fincas fue de
8413 insectos. Siendo la finca Las Vegas la de mayor número de insectos con 4365 en total, mientras que en la finca Linda Vista, se encontraron 4048 insectos.

En un estudio realizado por Gómez (2011), encontró una abundancia total de 3825 insectos en trampas de caída libre y galones en el cultivo de marañón en cambio en el cultivo de maracuyá con los mismos tipos de trampas, la abundancia total fue de 8413 insectos.

En 2014 Lacayo y Mayorga, relacionado con la investigación de maracuyá, en trampas de caída libre y galones en el cultivo de marango (Moringa oleifera), encontraron una abundancia total de 29152 insectos en cambio en el cultivo de maracuyá con el mismo tipo de trampa la abundancia total fue de 29152 insectos. Mairena (2015), en el cultivo de piña encontró una abundancia total de 10657 especímenes en trampas de caída libre y galones. Rugama y López (2011), en el cultivo de marañón, encontraron una abundancia total de 16064 insectos, en trampas de caída libre y galones.

Cuadro 1. Descripción, taxonomía y hábito alimenticio de insectos registrados en el cultivo de maracuyá, julio-noviembre 2016

\begin{tabular}{|c|c|c|c|c|c|}
\hline Orden & Familia & Género & Especie & $\begin{array}{c}\text { Nombre } \\
\text { común }\end{array}$ & $\begin{array}{c}\text { Hábito } \\
\text { alimenticio }\end{array}$ \\
\hline \multirow[t]{8}{*}{ Hymenoptera } & Anthophoridae & Xylocopa & $\mathrm{sp}$ & Abejorro & Nectarívoro \\
\hline & Formicidae & Atta & spp & Zompopo & Defoliador \\
\hline & Apidae & Apis & $\mathrm{sp}$ & Abeja & Nectarívoro \\
\hline & Halietidae & Halietus & sp & & \\
\hline & Apidae & Trigona & sp & Congo & Nectarívoro \\
\hline & Apidae & Apis & mellifera & Abeja & Polinivoro \\
\hline & Vespidae & Polistes & sp & Catalá & Depredador \\
\hline & Vespidae & Polybia & sp & Avispa & Depredador \\
\hline \multirow[t]{7}{*}{ Díptera } & Lonchaeidae & Dasiops & inedulis & $\begin{array}{l}\text { Mosca del } \\
\text { botón } \\
\text { floral }\end{array}$ & Fitófago \\
\hline & Agromyzidae & - & - & Mosca & ------ \\
\hline & Drosophilidae & Drosophila & melanogaster & $\begin{array}{l}\text { Mosca de } \\
\text { vinagre }\end{array}$ & Saprófago \\
\hline & Calliphoridae & Cochliomia & sp & $\begin{array}{l}\text { Barrenador } \\
\text { del ganado }\end{array}$ & Necrófago \\
\hline & Muscidae & Musca & domestica & Mosca & Saprófago \\
\hline & Tachinidae & Lespesia & sp & Mosca & Parasitoide \\
\hline & Syrphidae & Allograpta & sp & Mosca & $\begin{array}{l}\text { Nectarívoro } \\
\text { depredador }\end{array}$ \\
\hline \multirow[t]{8}{*}{ Hemíptera } & Coccidae & Ceroplastes & cirripediformes & Escama & Fitófago \\
\hline & Pentatomidae & Nezara & viridula & Chinche & Omnívoro \\
\hline & Pentatomidae & Euschistus & sp & $\begin{array}{l}\text { Chinche } \\
\text { marrón }\end{array}$ & Fitófago \\
\hline & Membracidae & Umbonia & $\mathrm{sp}$ & Chinche & Zoófago \\
\hline & Cicadelidae & - & - & Chicharrita & Fitófago \\
\hline & Membracidae & Umbonia & crassicornis & $\begin{array}{l}\text { Torito } \\
\text { verde }\end{array}$ & Fitófago \\
\hline & Fulgoridae & - & - & Chicharrita & Fitófago \\
\hline & Coreidae & Leptoglossus & zonatus & Chinche & Fitófago \\
\hline \multirow[t]{5}{*}{ Lepidóptera } & Noctuidae & Spodoptera & $\mathrm{sp}$ & Palomillas & Nectarivoro \\
\hline & Noctuidae & Mocis & latipes & $\begin{array}{l}\text { Gusano } \\
\text { medidor }\end{array}$ & Fitófago \\
\hline & Pyralidae & Rupela & $\mathrm{sp}$ & Mariposa & Nectarívoro \\
\hline & Nymphalidae & Anarthia & fatima & Palomillas & Nectarivoro \\
\hline & Piéridae & Phoebis & $\mathrm{sp}$ & Mariposa & Nectarívoro \\
\hline \multirow[t]{2}{*}{ Orthoptera } & Gryllidae & Gryllus & $\mathrm{sp}$ & Grillo & Defoliador \\
\hline & Tettigonidae & Conocephalus & sp & Esperanza & Defoliador \\
\hline \multirow[t]{12}{*}{ Coleóptera } & Scarabaeidae & Cotinis & sp & Escarabajo & Saprófago \\
\hline & Scarabaeidae & Melanarius & pterostichus & Escarabajo & Saprófago \\
\hline & Tenebrionidae & Epitragus & sp & Escarabajo & Saprófago \\
\hline & Scarabaeidae & phyllophaga & sp & $\begin{array}{l}\text { Gallina } \\
\text { ciega }\end{array}$ & Fitófago \\
\hline & Elateridae & Aeolus & $\mathrm{sp}$ & Escarabajo & Saprófago \\
\hline & Coccinelidae & Coccinella & sp & Escarabajo & Depredador \\
\hline & Chrysomelidae & Diabrotica & speciosa & Escarabajo & Fitófago \\
\hline & Elateridae & Conoderus & sp & Escarabajo & Saprófago \\
\hline & Chrysomelidae & Chrysolina & fastuosa & Escarabajo & Fitófago \\
\hline & Curculionidae & Rhynchoporus & ferrugineus & Escarabajo & Frugívoro \\
\hline & Chrysomelidae & Deloyala & $\mathrm{sp}$ & Escarabajo & Fitófago \\
\hline & Cerambycidae & Lagoherios & sp & Escarabajo & Xilófago \\
\hline Dermáptera & Forficulidae & Dorus & $\mathrm{sp}$ & Tijereta & Depredador \\
\hline Odonata & $\begin{array}{l}\text { Coenagrionidae } \\
\text { Libellulidae }\end{array}$ & $\begin{array}{l}\text { Coenagrion } \\
\text { Libellula }\end{array}$ & $\begin{array}{l}\text { sp } \\
\text { luctuosa }\end{array}$ & $\begin{array}{l}\text { Pipilacha } \\
\text { Pipilacha }\end{array}$ & $\begin{array}{l}\text { Depredador } \\
\text { Depredador }\end{array}$ \\
\hline
\end{tabular}


Abundancia total de insectos por tipo de trampa. En el Cuadro 2, se presenta la abundancia total de insectos El total de insectos en ambas fincas fue de 8413 , siendo la finca Las Vegas la que presentó mayor número de insectos, en las trampas de caída libre fue de 1991 insectos, y en trampas de galones fue 2374 , mientras que en la finca Linda Vista en trampas de caída libre fue de 1702 insectos, y en galones 2346 en total, siendo mejor las trampas de galones que capturaron más insectos debido a que probablemente es porque la melaza es un atrayente para los insectos voladores, ya que el olor y el sabor dulce es apetecido para estos.

Cuadro 2. Abundancia total de insectos por finca y tipe de trampa.

\begin{tabular}{lccc}
\hline \multicolumn{1}{c}{ Fincas } & Trampas de caída libre & Trampas de galones & Total \\
\hline Las Vegas & 1991 & 2374 & 4365 \\
Linda Vista & 1702 & 2346 & 4048 \\
\hline Total & 3693 & 4720 & 8413 \\
\hline
\end{tabular}

Abundancia de insectos por tipo de orden. Los principales ordenes de insectos encontrados fueron Díptera, Hymenoptera, Coleóptera, Lepidóptera y Hemíptera (Cuadro 3). Al comparar la abundancia de estos cinco órdenes de insectos, se observó que existió mayor cantidad de insectos por orden en la finca Las Vegas con tres órdenes y solo dos órdenes en la finca Linda Vista. El total de insectos encontrados por cada orden, en la finca Las Vegas fue Diptera con 1 202, Hymenoptera 695, Coleóptera 532, Lepidoptera 286 y Hemiptera 61, mientras el número de insectos por orden en la finca Linda Vista fue para Diptera de 2 063, Hymenoptera 706, Coleoptera 507, Lepidoptera 236 y Hemiptera con 39 insectos, el orden con mayor cantidad de insectos presentados fue el Diptera.

Los dípteros son insectos de habito alimenticio: saprófago, nectarívoro y depredadores (Andrews y Quezada, 1989), (Jiménez - Martínez 2009).

Algunas larvas de la mosquita del botón floral, fueron encontradas haciendo daño, por lo tanto el orden Díptera se considera de importancia económica en el cultivo de la maracuyá, ya que estas larvas causan la caída de los botones florales y pueden afectar el rendimiento del cultivo y causar pérdidas económicas al productor.

Los insectos del orden Hymenoptera como los de la familia Apidae y Vespidae, son polinizadores, producen miel, son agentes de control biológico de plagas en los cultivos. Los insectos del Coleoptera tienen hábitat y alimentación variables ejemplo Scarabidae, Chrysomelidae y Coccinelidae, pueden ser (minadores, barrenadores, trozadores, defoliadores, predadores entre otros), También son plagas de cultivos, predadores de plagas y malezas, participan en reciclaje de la materia orgánica. Lepidoptera ejemplo la familia Noctuidae y Pyralidae son larvas generalmente fitófagas (mayormente fitófago externos y pocos minadores de hojas), son predadores y parasitas. Reciben nombres como cortadores, trozadores, soldados, medidores y barrenadores. Hemiptera ejemplo la familia Coreidae, Coccidae y Fulgoridae la mayoría son fitófagos se alimentan principalmente de la savia de las plantas, algunos son depredadores de plagas y malezas (Sáenz y De la Llana, 1990).

Se encontró que los dos órdenes con mayor abundancia de insectos en las fincas son Diptera (en algunos casos son plagas cómo la mosquita del botón floral, plaga principal de la maracuyá) e Hymenoptera (polinizadores en su mayoría, como el Xyloscopa y Apis que son los que se encuentran polinizando la flor de la maracuyá), por eso son los órdenes de insectos más abundantes.

Cuadro 3. Abundancia de insectos por finca y de orden

\begin{tabular}{lccc}
\hline \multirow{2}{*}{$\begin{array}{l}\text { Órdenes de } \\
\text { insectos }\end{array}$} & \multicolumn{3}{c}{ Número de insectos por finca } \\
\cline { 2 - 4 } & $\begin{array}{c}\text { Fincas Las } \\
\text { Vegas }\end{array}$ & $\begin{array}{c}\text { Finca Linda } \\
\text { Vista }\end{array}$ & Total \\
\hline Diptera & 1202 & 2063 & 3265 \\
Hymenoptera & 695 & 706 & 1401 \\
Coleoptera & 532 & 507 & 1039 \\
Lepidoptera & 286 & 236 & 522 \\
Hemiptera & 61 & 39 & 100 \\
\hline Total & 2776 & 3551 & 6327 \\
\hline
\end{tabular}

Comparación de la abundancia de insectos de las familias. Se comparó la abundancia de insectos por familia encontrados en el cultivo de maracuyá en las fincas (Cuadro 4). Se observó que hubo mayor cantidad de insectos por familia en la finca Las Vegas en ocho familias de las 12 mencionadas y solo en cuatro de 12 en la finca Linda Vista.

La familia más abundante en este caso fue la Drosophilidae, encontrándose el mayor número de insectos en la finca Linda Vista con 1869 y siendo menor Las Vegas con 1377 insectos, esta familia fue constante, presentando los valores más altos durante todas las fechas de colecta. Respecto a sus hábitos alimenticios se sabe que los insectos de esta familia son saprófagos descomponedores de materia orgánica, estos estaban presentes en ambas fincas debido a que se encontraban muchos frutos maduros caídos en el suelo, esto favoreció a la aparición de estos insectos, además probablemente fueron atraídos por la melaza.

En este estudio, la familia Formicidae presentó un mayor número de individuos, encontrándose el mayor número de insectos en la finca Linda Vista con 458 y siendo menor Las Vegas con 451 insectos, esta familia fue constante en todas las fechas de colecta, la familia Noctuidae fue la tercera más abundante, encontrándose el mayor número de insectos en la finca Las Vegas con 298 y siendo menor Linda 
Vista con 231 insectos, esta familia se presentó en todas las fechas de colecta.

Mairena (2015), encontró una abundancia total de insectos de las familias Formicidae de 4 321, Noctuidae 286, Apidae 202 y Elateridae 374 insectos, en trampas de caída libre y galones, mientras que en este estudio se encontró una abundancia de insectos de la familia Formicidae de 909, Apidae 235 y Elateridae de 45 insectos.

Cuadro 4. Abundancia de familias de insectos por finca

\begin{tabular}{|c|c|c|c|}
\hline \multirow[b]{2}{*}{ Familia } & \multicolumn{3}{|c|}{ Familias de insectos capturados por finca } \\
\hline & Las Vegas & Linda Vista & Total \\
\hline Drosophilidae & 1377 & 1869 & 3246 \\
\hline Formicidae & 451 & 458 & 909 \\
\hline Noctuidae & 298 & 231 & 529 \\
\hline Tenebrionidae & 222 & 296 & 518 \\
\hline Curculionidae & 273 & 193 & 466 \\
\hline Pachyboloidae & 283 & 144 & 427 \\
\hline Eunicidae & 223 & 81 & 304 \\
\hline Lonchaidae & 191 & 83 & 274 \\
\hline Apidae & 130 & 105 & 235 \\
\hline Chrysopidae & 135 & 69 & 204 \\
\hline Vespidae & 85 & 118 & 203 \\
\hline Scolitydae & 90 & 51 & 141 \\
\hline Muscidae & 82 & 54 & 136 \\
\hline Pentatomidae & 55 & 36 & 91 \\
\hline Chrysomelidae & 65 & 21 & 86 \\
\hline Gryllidae & 40 & 21 & 61 \\
\hline Forfuculidae & 36 & 16 & 52 \\
\hline Helicidae & 31 & 20 & 51 \\
\hline Elateridae & 23 & 22 & 45 \\
\hline Tachinidae & 29 & 16 & 45 \\
\hline Membracidae & 21 & 23 & 44 \\
\hline Limacidae & 23 & 20 & 43 \\
\hline Agromyzidae & 22 & 19 & 41 \\
\hline Pentatomidae & 25 & 11 & 36 \\
\hline Tettigonidae & 22 & 6 & 28 \\
\hline Coenagrionidae & 11 & 15 & 26 \\
\hline Calliphoridae & 11 & 13 & 24 \\
\hline Coreidae & 17 & 5 & 22 \\
\hline Anthophoridae & 12 & 6 & 18 \\
\hline Fulgoridae & 12 & 4 & 16 \\
\hline Coccinelidae & 9 & 1 & 10 \\
\hline Cicadelidae & 3 & 1 & 4 \\
\hline
\end{tabular}

Riqueza total de familias de insectos. La riqueza total fue de 32 familias de insectos, siendo la finca Las Vegas, la que mayor riqueza presentó con 32, comparada con la finca Linda Vista con 31 familias encontradas.

Lacayo y Mayorga (2014), usando trampas de caída libre y galones en el cultivo de marango, encontraron una riqueza de 15 familias de insectos, en el presente estudio de maracuyá fue de 32 familias de insectos.

En el cultivo de marañón Rugama y López (2011), utilizando igual tipo de trampa encontraron una riqueza total de 35 familias de insectos.

Riqueza total de géneros de insectos. La riqueza total de géneros de insectos, en ambas fincas fue de 41 , siendo la finca Las Vegas, la que mayor riqueza presentó con 41 géneros de insectos, comparada con la finca de Linda Vista con 39 géneros de insectos encontrados.
La plantación de maracuyá en la finca Las Vegas es de mayor edad y probablemente esto influye en la presencian de insectos; otro factor que no descartamos es la aplicación moderada de químicos y en cambio en la finca Linda Vista es severa.

En el cultivo de marango Lacayo y Mayorga (2014), en trampas de caída libre y galones encontraron una riqueza total de 160 géneros de insectos, en el presente estudio de maracuyá utilizando el mismo tipo de trampas, la cantidad fue menor, encontrando 41 géneros.

Índice de diversidad de Shannon-Weaver. Shannon y Weaver (1949), definen la diversidad cómo el número de especies existentes dentro de un mismo ecosistema. La diversidad de un ecosistema depende de tres factores, el número de especies presente, la composición del paisaje y las interacciones que existen entre las diferentes especies llegando a un equilibrio demográfico entre ellas. El índice de Shannon-Weaver, se usa en ecología u otras ciencias similares para medir la biodiversidad específica. Este índice se representa normalmente como $\mathrm{H}$ y se expresa con un número positivo, que en la mayoría de los ecosistemas naturales varía entre 0,5 y 5 , aunque su valor normal está entre 2 y 3 ; valores inferiores a 2 se consideran bajos y superiores a 3 son altos.

En este estudio, el promedio de índice de diversidad fue ligeramente mayor en la finca Las Vegas (1.09), en comparación a la finca Linda Vista (1.07). Al comparar la diversidad entre familias de insectos, en la finca Las Vegas, la diversidad fue entre 1.44 y 1.00 , siendo 1.44 para la familia Drosophilidae y el 1.00 para la familia Cicadelidae, mientras que, en la Finca Linda Vista, el índice de diversidad de la familia Drosophilidae fue de 1.43 y 1.00 para la familia Cicadelidae.

En un estudio realizado por Téllez y Jirón (2014), en el cultivo de marango, encontraron un promedio de índice de diversidad de 1.14 y 1.13 en trampas de caída libre y galones en dos fincas, índice similar al promedio encontrado en la finca Linda Vista y la finca Las Vegas.

En 2014 Lacayo y Mayorga en el cultivo de marango, encontraron que el de índice de diversidad fue de 1.13 y 1.12 en trampas de caída libre y galones en dos parcelas, parecido al promedio encontrado en este estudio que fue de 1.09 y 1.07 .

Distribución temporal de la familia Coccidae. Se muestra la distribución temporal de insectos de la familia Coccidae en ambas fincas, desde el 8 de julio hasta el 25 de noviembre (Figura, 1), registrándose insectos de esta familia solo en Linda Vista, en ocho de 21 fecha de colecta, los mayores picos poblacionales fueron el 19 de agosto con 13 insectos, 14 de octubre 18 insectos y el 4 de noviembre 16 insectos colectados. Se puede especular que la variación temporal de 
Cuadro 5. Índice de diversidad Shannon-Weaver de las principales familias de insectos por finca

\begin{tabular}{|c|c|c|}
\hline \multirow{2}{*}{$\begin{array}{c}\text { Familias de } \\
\text { insectos }\end{array}$} & \multicolumn{2}{|c|}{ Índice de diversidad de Shannon-Weaver } \\
\hline & Las Vegas & Linda Vista \\
\hline Drosophilidae & 1.44 & 1.43 \\
\hline Formicidae & 1.26 & 1.28 \\
\hline Noctuidae & 1.20 & 1.18 \\
\hline Tenebrionidae & 1.16 & 1.21 \\
\hline Curculionidae & 1.19 & 1.16 \\
\hline Pachyboloidae & 1.19 & 1.13 \\
\hline Eunicidae & 1.16 & 1.08 \\
\hline Lonchaeidae & 1.14 & 1.08 \\
\hline Apidae & 1.11 & 1.10 \\
\hline Chrysopidae & 1.11 & 1.07 \\
\hline Vespidae & 1.08 & 1.11 \\
\hline Scolitydae & 1.08 & 1.06 \\
\hline Muscidae & 1.08 & 1.06 \\
\hline Pentatomidae & 1.06 & 1.04 \\
\hline Chrysomelidae & 1.06 & 1.03 \\
\hline Gryllidae & 1.04 & 1.03 \\
\hline Forficulidae & 1.04 & 1.02 \\
\hline Helicidae & 1.03 & 1.03 \\
\hline Elateridae & 1.03 & 1.03 \\
\hline Tachinidae & 1.03 & 1.02 \\
\hline Membracidae & 1.03 & 1.03 \\
\hline Limacidae & 1.03 & 1.03 \\
\hline Agromyzidae & 1.03 & 1.03 \\
\hline Pentatomidae & 1.03 & 1.02 \\
\hline Tettigonidae & 1.03 & 1.01 \\
\hline Coenagrionidae & 1.01 & 1.02 \\
\hline Calliphoridae & 1.01 & 1.02 \\
\hline Coreidae & 1.02 & 1.01 \\
\hline Anthophoridae & 1.02 & 1.01 \\
\hline Fulgoridae & 1.02 & 1.01 \\
\hline Coccinelidae & 1.01 & 1.00 \\
\hline Cicadelidae & 1.00 & 1.00 \\
\hline Promedio & 1.09 & 1.07 \\
\hline
\end{tabular}

esta familia en la finca Linda Vista se vio debido a la edad del cultivo ya que estaba terminando su ciclo productivo y probablemente por existir debilitamiento en las plantas, ademas no se realizaba deshoje debido a que la enramada estaba cubierta totalmente, esto la convirtió más apetecida por las escamas de cera al encontrar las condiciones para alimentarse y reproducirse.

Los Coccidae o Lecaniidae son escamas redondas, gruesas, a veces un poco alargada. El exoesqueleto es duro y cubierto de cera. La hembra tiene patas en general no tienen antenas y los machos son alados o ápteros según las especies. Esta familia está distribuida desde Asia, Estados unidos, Cuba, Bermuda, Puerto Rico, México, Belice, Guatemala, Honduras y Nicaragua (Maes, 1998).

Las escamas son de tamaño pequeño (1-8 $\mathrm{mm})$, cuerpo generalmente ovoide, aplanado, y algunas veces hemisférico, con un exoesqueleto duro y liso, antenas ausentes, aparato bucal con dos segmentos, patasgeneralmente presentes, ciclo de vida muy complejo. La ninfa en el primer estadio posee antenas y patas y es muy activa. Al segundo estadio en muchas especies pierde las patas y antenas y se cubre de una escama de cera. Estos insectos son plagas de cultivos (Sáenz y De la Llana, 1990). En el cultivo de maracuyá se encontró a la escama (Ceroplastes cirripediformes) en las guías y algunas en las hojas succionando la savia de este.

Según Jiménez-Martínez (2009), las escamas tienen importancia económica debido a que pueden atacar fuertemente a la planta llegando a debilitarlas y a defoliar, también producen mielecilla.

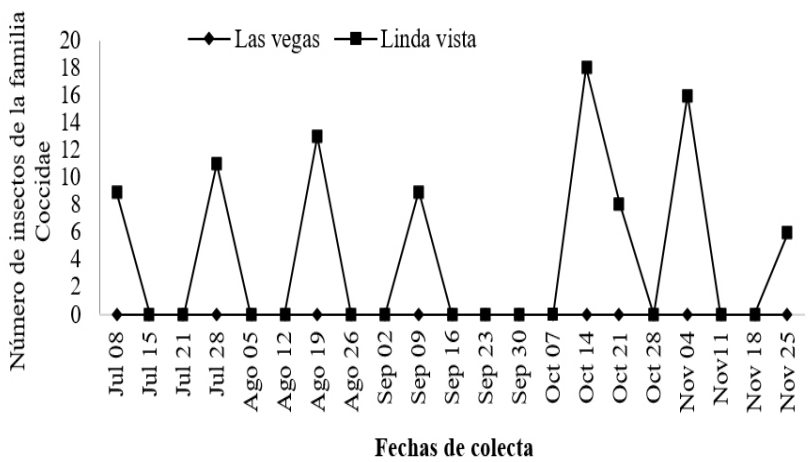

Figura 1. Distribución temporal de la familia Coccidae en el cultivo de maracuyá entre julio a noviembre 2016.

Distribución temporal de la familia Coreidae. Se registró mayor población de insectos de esta familia fue en la finca Las Vegas en seis de 21 fechas de colecta, en comparación con la finca Linda Vista donde se encontró en tres de 21 fechas de colectas. Las fechas que presentaron los mayores picos poblacionales en la finca Las Vegas fue el 14 de octubre con cinco insectos, el 11 de noviembre tres insectos y el 18 de noviembre cuatro insectos, mientras que en la finca Linda Vista los mayores picos poblacionales fueron el 23 de septiembre con dos insectos y el 30 se septiembre con dos insectos colectados.

Téllez y Jirón (2014), en un estudio realizado en el cultivo de marango en trampas de galón, encontraron dos insectos promedio por trampa, mientras en este estudio en el cultivo de maracuyá, se encontró un promedio de 1.09 insectos.

Los Coreidae son los más grandes de la superfamilia, miden de 7 a $40 \mathrm{~mm}$. Sonfitófagos, se alimentan principalmente de savia y frutas pero algunos comen semillas (Maes, 1998), están distribuidos desde Estados unidos, Grenada, México, Guatemala, Belice, Honduras, El Salvador y Nicaragua.

Las chinches de la familia Coreidae presentan un tamaño de mediano a grande, llegando algunas especies hasta $30 \mathrm{~mm}$ de longitud, su cuerpo oval-alargada y generalmente robusto, la membrana de los hemélitros presentan de 20 venas longitudinales, glándulas odoríferas entre el segundo y tercer par de patas, tibia posterior algunas veces dilatadas. Adultos y ninfas succionan savia de los brotes tiernos, tallos, hojas y frutas, algunas especies prefieren alimentarse de semillas y otras pocas son depredadoras (Nunes y Dávila, 2004).

En la familia Coreidae, casi todos son fitófagos. Entre las especies más comunes se encuentran las chinches 
con patas laminadas, chinches hediondas y las chinches patas de hoja (Andrews y Caballero, 1989).

Las Coreidae son insectos de mediano a grande (10-40 mm), cuerpo alargado, robusto, color, generalmente oscuro, gris, café o negro, cabeza más pequeña y estrecha que el pronoto, antenas con cuatro segmentos, alas delanteras con más de siete venas longitudinales en la parte membranosa, glándulas odoríferas entre el segundo y tercer pares de patas. La mayoría son fitófagos; algunos son predadores. Son plagas de importancia económica de cultivos, entre ellas tenemos a Leptoglosus zonatus Dallas (chinche patas de hojas) (Sáenz y De la Llana, 1990), (Jiménez- Martínez, 2009). En este estudio, se encontraron estos insectos en las trampas de galón.

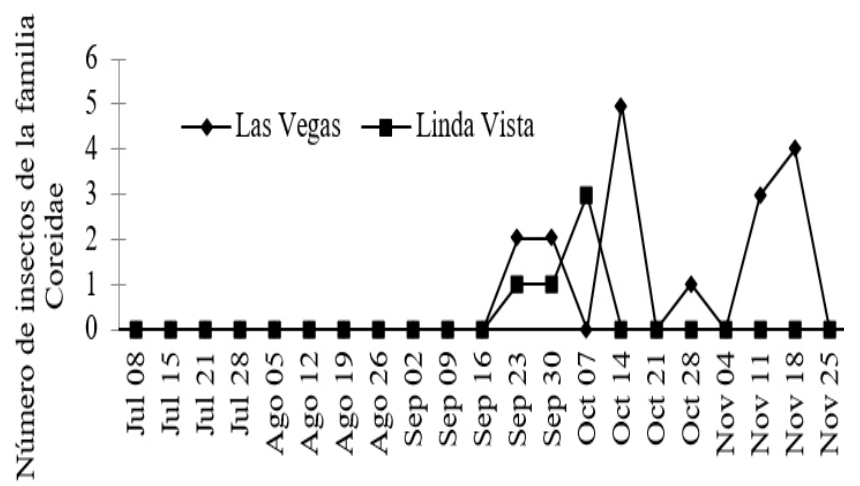

\section{Fechas de colecta}

Figura 2. Distribución temporal de la familia Coreidae en el cultivo de maracuyá entre Julio a Noviembre del 2016.

Distribución temporal de la familia Anthophoridae. Existió mayor población de insectos de esta familia en la finca Las Vegas en cinco de las 21 fechas de colecta, en comparación con la finca Linda Vista con solo 4 de 21 fechas de colecta, los mayores picos poblacionales en la finca Linda Vista fueron el 23 de septiembre con dos insectos, el 21 de octubre dos insectos, el 28 de octubre tres insectos, el 4 de noviembre dos insectos y el 25 de noviembre cuatro insectos, fueron el 19 de agosto con dos insectos y el 30 de septiembre dos insectos colectados.

La familia Anthophoridae llamada Apidae en la investigación de (Téllez y Jirón 2014), y llamada por Maes (1998), Anthophoridae hace referencia al mismo género Xylocopa.

Los Anthophoridae forman una familia muy amplia, muy variada de abejas robustas y peludas. Hacen sus nidos en el suelo. Ceratina y Xylocopa son considerados cómo una subfamilia separada (Xylocopinae), este grupo hace su nido en el interior de las ramas (Maes, 1998), Están distribuidos en Estados unidos, México y Nicaragua (León, Managua, Masaya y en la Isla de Ometepe).

Los Anthophoridae son de tamaño pequeño a grande (3-20 mm), expansión alar (5-35 mm) cuerpo delgado o robusto; color y pubescencia variable, metatibias con espinas apicales, abejas solitarias con nidos en el suelo (Anthophorinae,) o en madera (Xylocopinae) algunas de estas especies hacen nidos dentro de la madera de edificios ejemplo de ellas (Xylocopa sp) (Sáenz y De la Llana, 1990). En maracuyá se encontraron los Xylocopa sp en las trampas de galón, estos insectos son los principales polinizadores en este cultivo y fueron observados polinizando las flores en ambas fincas, principalmente en las primeras horas de la mañana.

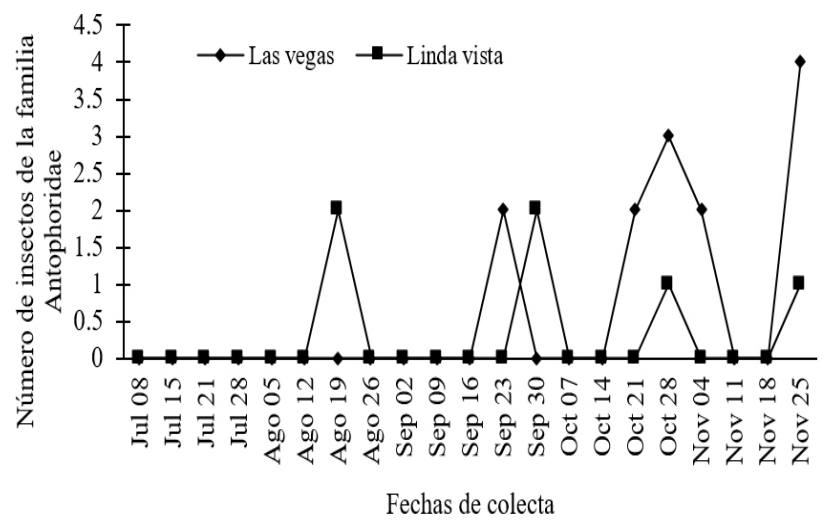

Figura 3. Distribución temporal de la familia Anthophoridae en el cultivo de maracuyá de julio a noviembre del 2016.

Distribución temporal de la familia Formicidae. En ambas fincas se encontró presencia de insectos en 19 de 21 fechas de colecta, los mayores picos poblacionales para la finca Linda Vista fueron el 30 de septiembre con 42 insectos, el 21 de octubre 53, el 28 de octubre 54, el 4 de noviembre 42 y el 11 de noviembre 47 insectos, mientras en Las Vegas, los mayores picos poblacionales fueron el 14 de octubre 45, el 21 de octubre 45 y el 28 de octubre 47 insectos.

En un estudio realizado por Estrada y Rodriguez (2009) mencionan que uno de los principales mecanismos de defensa de las pasifloras contra herbívoros consiste en atraer hormigas mediante la producción de néctar de glándulas extra florales (laminas foliares, peciolos, estipulas y brácteas florales). Este néctar contiene grandes cantidades de azucares y es rico en aminoácidos, lo cual explica la atracción para muchos insectos especialmente hormigas.

Rugama y López (2011), en el cultivo de marañón, con trampas de caída libre, se registraron 599 y 202 insectos promedio por trampa de la familia Formicidae en dos fincas, mientras en este estudio con el mismo tipo de trampa se encontró 451 y 458 insectos por finca.

Según Maes (1998), los Formicidae u hormigas forman una amplia familia que se encuentran en todo tipo de medios. Viven en sociedades donde encontramos individuos de diferentes castas, las principales son reproductores hembras y machos, soldados y obreros. Las colonias varían mucho según las especies desde docena de individuos hasta 
una docena de miles. Las colonias pueden ser de muchos tipos, principalmente en cavidades bajo la tierra en plantas.

La familia Formicidae es muy diversa en sus hábitos alimenticios. Esenrealidad, talvezlamásvariada enesteaspecto de todas las familias de insectos. Hay cazadores, saprófitos, fitófagos, y fungivoros. Ciertas especies matan plagas mientras que otras cuidan insectos dañinos. Hay especies que se alimentan de follaje, otras cortan el follaje para criar hongos.

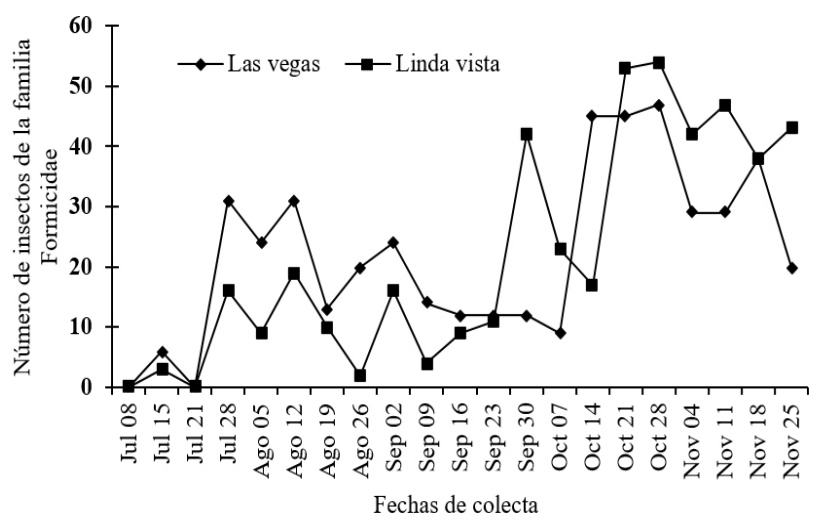

Figura 4. Distribución temporal de la familia Formicidae en el cultivo de maracuyá julio a noviembre del 2016.
Ciertas especies se especializan en alimentarse con sustancias grasosas, mientras que otras se alimentan únicamente de sustancias dulces, entre ellas tenemos zompopos, hormigas bravas, hormigas cosechadoras, hormigas carpinteras y hormigas esclavistas (Andrews y Caballero, 1989).

\section{CONCLUSIONES}

La mayor abundancia y riqueza de insectos asociados al cultivo de maracuyá, ocurrió en la finca Las Vegas. En cuanto a la riqueza de insectos se encontraron 36 familias, con 44 géneros, siendo los más numerosos los pertenecientes a las familias Apidae, Vespidae, Noctuidae, Pentatomidae y Scarabaeidae. En la distribución temporal, las familias más importantes fueron, las Lonchaeidae, Anthophoridae, Coccidae y Formicidae.

Se identificó un total de 80 familias de insectos, siendo las dos familias más dominantes Drosophilidae y la Formicidae en la finca Linda Vista, aunque de manera general la mayor dominancia fue para la finca Las Vegas.

Los mayores picos poblacionales para las familias Lonchaeidae, Coreidae, Coccidae, Anthophoridae y Formicidae, fueron entre octubre y noviembre.

\section{REFERENCIAS BIBLIOGRÁFICAS}

Andrews Keith. L., y Caballero, R. (1989). Guia para el estudio de órdenes y familias de insectos de Centroamérica. Francisco Morazán, Honduras: Escuela Agrícola Panamericana El Zamorano.

Andrews Keith. L., y Quezada, J. R. (1989). Manejo integrado de plagas insectiles en la Agricultura: Estado actual y futuro. Francisco Morazán, Honduras: Escuela Agrícola Panamericana El Zamorano.

Bolaños. R. (1997). Maracuyá cálala parchita. Managua, Nicaragua: CETREX. 122 p.

Estrada, A, y Rodriguez, A. (2009) Flores de pasión de Costa Rica, Editorial INBIo, 448 p.

Gerencia Regional Agraria La Libertad. (2009). "El cultivo del maracuyá” Passiflora edulis Sims forma. Flavicarpa. http://www. agrolalibertad.gob.pe/sites/default/files/MANUAL\%20DEL\%20CULTIVO\%20DE\%20MARACUYA 0.pdf

Gómez Martínez. J. (2011). Entomofauna y patógenos asociados al cultivo de marañón (Anacardium occidentale L.), en León, Nicaragua, entre los meses de julio 2009 a marzo 2010. (Tesis de maestría). http://repositorio.una.edu.ni/2167/1/tnh10g633p.pdf

INETER (Instituto Nicaragüense de Estudios Territoriales). (2016). Datos del municipio de Sébaco. biblioteca.enacal.com.ni/bibliotec/ Libros/enacal//0001/CD001005/Cap01.pdf

INTA (Instituto Nicaragüense de Tecnología Agropecuaria). (1996). Cultivo del maracuyá. Managua, Nicaragua.

Jiménez Martínez, E. S. (2009). Texto Básico: Entomología. Managua Nicaragua: UNA.

Lacayo Rodríguez, R. T., y Mayorga Mendoza, J. R. (2014). Abundancia, riqueza y diversidad insectil asociada al cultivo de moringa (Moringa oleifera L.) en Managua, Nicaragua, Entre los meses de mayo a diciembre del 2013. (Tesis de grado). http://repositorio. una.edu.ni/3156/1/tnh101129a.pdf

Laguna González, T. J., Flores Téllez, E. R., Pérez Siles, A. R., Martínez Matamoros, M. A., Escoto Mayorga, S. E., y Castillo Urbina, J. A. (2015). Guía técnica del cultivo de maracuyá. Matagalpa, Nicaragua: CRS-CARITAS Matagalpa.

López González, J. M. (2002). Cultivo del Maracuyá. Mangua, Nicaragua: UNA-Nicaragua

Maes, J. M. (1998). Insectos de Nicaragua: catálogo de los insectos y artrópodos terrestres de Nicaragua. León, Nicaragua.

Mairena Vásquez, C. L. (2015). Identificación y fluctuación poblacional de insectos asociados al cultivo de la piña (Ananas comusus L. Merril.), en Ticuantepe, Nicaragua, Entre los meses de marzo a septiembre 2014. (Tesis de maestría). http://repositorio.una.edu. $\mathrm{ni} / 3363 / 1 / \mathrm{tnh} 10 \mathrm{~m} 228 . \mathrm{pdf}$

Nunes Zuffo, C., y Dávila Arce, M. L. (2004). Taxonomía de las Principales Familias y Subfamilias de Insectos de interés Agrícolas en Nicaragua. Estelí, Nicaragua: UCATSE. 
Rugama Lovo, I. M., y Lopez Vílchez, M. E. (2011). Identificación y descripción de los principales insectos rastreros asociados al cultivo de marañón (Anacardium occidentale L.), orgánico y convencional en León, Nicaragua, durante los meses de agosto 2009 a marzo 2010. (Tesis de grado). http://repositorio.una.edu.ni/2142/1/tnh10r928i.pdf

Sáenz, M., y De La Llana, A. (1990). Entomología sistemática. Managua, Nicaragua: UNA.

SAS Institute, (2003). Serial Attached SCS. North Caroline: Estados Unidos: University of Nebraska.

Shannon, C. E y Weaver, W. (1949). The mathematical theory of communication. Illinois, Estados Unidos: University of Illinois Press.

Téllez Manzanares, M. S., y Jirón Cortez, V. M. (2014). Identificación y variación poblacional de insectos asociados al cultivo de marango (Moringa oleifera L.) en Managua, Nicaragua durante los meses de noviembre 2012 a abril 2013. (Tesis de grado). http:// repositorio.una.edu.ni/2756/1/tnh10t275i.pdf 